\title{
Evolution of long water waves in variable channels
}

\author{
By MICHELLE H. TENG $\uparrow$ AND THEODORE Y. WU \\ Engineering Science, 104-44, California Institute of Technology, Pasadena, CA 91125, USA
}

(Received 1 September 1992 and in revised form 16 November 1993)

This paper applies two theoretical wave models, namely the generalized channel Boussinesq (gcB) and the channel Korteweg-de Vries (cKdV) models (Teng \& Wu 1992) to investigate the evolution, transmission and reflection of long water waves propagating in a convergent-divergent channel of arbitrary cross-section. A new simplified version of the $\mathrm{gcB}$ model is introduced based on neglecting the higher-order derivatives of channel variations. This simplification preserves the mass conservation property of the original gcB model, yet greatly facilitates applications and clarifies the effect of channel cross-section. A critical comparative study between the $\mathrm{gcB}$ and $\mathrm{cKdV}$ models is then pursued for predicting the evolution of long waves in variable channels. Regarding the integral properties, the gcB model is shown to conserve mass exactly whereas the $\mathrm{cKdV}$ model, being limited to unidirectional waves only, violates the mass conservation law by a significant margin and bears no waves which are reflected due to changes in channel cross-sectional area. Although theoretically both models imply adiabatic invariance for the wave energy, the gcB model exhibits numerically a greater accuracy than the $\mathrm{cKdV}$ model in conserving wave energy. In general, the gcB model is found to have excellent conservation properties and can be applied to predict both transmitted and reflected waves simultaneously. It also broadly agrees well with the experiments. A result of basic interest is that in spite of the weakness in conserving total mass and energy, the $\mathrm{cKdV}$ model is found to predict the transmitted waves in good agreement with the $\mathrm{gcB}$ model and with the experimental data available.

\section{Introduction}

Evolutions of nonlinear long waves, such as a tsunami wave climbing up a threedimensional ocean shelf or a flood wave propagating in a river with variable crosssection, are problems of fundamental importance, both theoretically and in practice because of environmental concerns. For such problems, the classical Boussinesq model has been generalized for application to shallow water of gradually varying depth in two horizontal dimensions by Peregrine (1967) and for forced waves by Wu $(1979,1981)$. The Boussinesq models conserve mass and can be applied to evaluate waves propagating in all horizontal directions and, simultaneously, the reflected waves. Applications of this family of models have been made by Chwang \& Wu (1976) to study self-focusing and reflection of cylindrical solitary waves over radially varying topographies and by Schember (1982) to simulate solitary waves climbing up concave or convex ocean shelves.

For modelling channel waves, the Korteweg-de Vries (KdV) model has been extended to study long waves in rectangular channels of variable depth and width by Shuto (1974) and other authors cited by Teng \& Wu (1992, hereinafter referred to as

$\dagger$ Present address: Department of Civil Engineering, University of Hawaii at Manoa, Honolulu, HI 96822, USA. 
TW1). Models of the KdV family, being derived for unidirectional waves only, have been noted to have the drawback of not conserving mass but have been found by Miles (1979) and others to be adiabatically invariant in energy. Shuto's model has been examined by Chang, Melville \& Miles (1979) and compared with their experiment on propagation and reflection of a solitary wave in a linearly divergent and linearly convergent rectangular channel of uniform depth. Despite the drawback of neglecting the reflected waves and of not conserving mass, Shuto's model was found to predict the transmitted waves in good agreement with the experiment. An explanation for the missing link between the fluid mass lost by the channel $\mathrm{KdV}$ model and the fairly wellpredicted transmitted wave which results from having shed the lost mass has been provided by Kirby \& Vengayil (1988) based on their set of coupled KdV equations. This set of coupled equations was constructed using a heuristic approach based on the method of operator correspondence and a linear representation of the reflected waves. Further variations of channel flow models include the Kadomtsev-Petviashvili (K-P) equation extended to variable topographies by Liu, Yoon \& Kirby (1985) and the Euler model adopted by Choi et al. (1990). The Green-Naghdi equations have been applied by Ertekin \& Wehausen (1986) to study waves travelling in variable rectangular channels.

This paper attempts to compare the two theoretical models, namely the generalized channel Boussinesq $(\mathrm{gcB})$ and the channel Korteweg-de Vries (cKdV) models presented in TW1, with respect to evolution, transmission and reflection of long water waves in convergent-divergent channels of arbitrary cross-section. In $\$ 2$, a new simplified version of the $\mathrm{gcB}$ model is derived by neglecting the higher-order derivatives of channel variations, which modifies, only very slightly, the strength of the dispersion effects, leaving the other terms in the equations intact. This simplification preserves the mass conservation property of the original gcB model, as is shown in $\S 4$. Numerically, the new version is found to agree, with very small differences, with the original model even for finite variations of channel geometry, as demonstrated in $\S 3$. In $\S 5$, typical numerical results are presented, and are compared with the experiment by Chang $\mathrm{et} \mathrm{al}$. (1979) in §6. From these comparative studies the gcB model is found to be broadly in good agreement with the experiment. Another result of interest is that despite the drawback of poor conservation of mass and neglect of reflected waves, the $\mathrm{cKdV}$ model is nevertheless found invariably to predict the transmitted waves in good agreement with the $\mathrm{gcB}$ model and the experiment.

\section{Theory}

\subsection{Model equations}

Generation and propagation of nonlinear long waves in a variable channel of arbitrary shape, whose width and water depth are supposed to be of the same order, can be described by the generalized channel Boussinesq (gcB) model (Teng \& Wu 1990, 1992):

where

$$
\begin{gathered}
(2 b \tilde{\zeta})_{t}+\left[\left(2 b \tilde{h}-A_{d}+2 b \tilde{\zeta} \bar{u}\right]_{x}=\left(A_{d}\right)_{t}\right. \\
\bar{u}_{t}+\bar{u} \bar{u}_{x}+\tilde{\zeta}_{x}+\psi r(x, t)=-\left(\widetilde{p_{a}}\right)_{x}
\end{gathered}
$$

and for unidirectional waves by the channel Korteweg-de Vries (cKdV) model:

$$
\frac{1}{c_{0}} \tilde{\zeta}_{t}+\left(1+\frac{3 \tilde{\zeta}}{2 \tilde{h}_{0}}\right) \tilde{\zeta}_{x}+\frac{1}{2} \psi+\left(\frac{\tilde{h}_{0 x}}{4 \tilde{h}_{0}}+\frac{b_{0 x}}{2 b_{0}}\right) \tilde{\zeta}=-\frac{1}{2}\left(\frac{1}{2 b_{0}} A_{d}+{\widetilde{p_{a}}}_{)_{x}},\right.
$$


with the tilde denoting the surface mean averaged across the channel surface width and the bar the section mean averaged over the cross-sectional area. All variables in (1)-(4) are written in non-dimensional form with the length variables scaled by the unperturbed mean water depth, $h_{c}$, and the time scaled by $\left(h_{c} / g\right)^{\frac{1}{2}}, g$ being the gravitational acceleration. (Note that in TW1, $x$ was scaled by $\lambda$ instead of $h_{c}$.) The two small parameters $\alpha=a / h_{c}$ and $\epsilon=h_{c} / \lambda$ satisfy $\epsilon \ll 1$ and $\alpha=O\left(\epsilon^{2}\right)$ with $a$ being a typical wave amplitude and $\lambda$ a typical wavelength. Here, $\tilde{\zeta}(x, t)$ denotes the mean free-surface elevation at station $x$ at time $t, \bar{u}(x, t)$ the section-mean velocity in the longitudinal $x$-direction, $b(x, t)=b_{0}(x)-b_{d}(x, t)$ the channel half-width at the free surface, $\tilde{h}(x, t)=$ $\tilde{h}_{0}(x)-\tilde{h}_{d}(x, t)$ the mean water depth, $A(x, t)=A_{0}(x)-A_{d}(x, t)+2 b \tilde{\zeta}$ the total crosssectional area (with $b_{0}, \tilde{h}_{0}$ and $A_{0}=2 b_{0} \tilde{h}_{0}$ being referred to the rest state) and $c_{0}$, defined as $c_{0}=\left(g \tilde{h}_{0}(x)\right)^{\frac{1}{2}}$, is the dimensionless critical wave speed. With $b_{d}(x, t)$ and $\tilde{h}_{d}(x, t)$ acting as forcing disturbances, $A_{d}=A_{0}-2 b \tilde{h}$ is the cross-sectional blockage ratio of these disturbances or of submerged moving variations of water depth and channel width in space.

Further, expression (3) relates the dispersion function $\psi(x, t)$ to the second-order term $\alpha^{2} \phi_{2}(x, y, z, t)$ of the original perturbation expansion for the velocity potential (TW1, equations (39)-(42)). As assumed, for finite-amplitude long waves generated by weak external forcings and propagating in a gradually variable channel, we have $\zeta$, $\bar{u}=O(\alpha), \tilde{\zeta}_{x}, \tilde{\zeta}_{t}, \bar{u}_{x}=O(\epsilon \alpha)$, and for the channel variation we assume, in addition, that $b_{t}, \tilde{h}_{t}=O\left(\epsilon \alpha^{2}\right), b_{x}, \tilde{h}_{x}=O(\epsilon \alpha)$, with $\alpha \ll 1, \epsilon \ll 1$, and $O(\alpha)=O\left(\epsilon^{2}\right)$. In TW1 for the original models, $b_{t}, \tilde{h}_{t}=O(\epsilon \alpha)$ for the gcB model and $b_{t}, \tilde{h}_{t}=O\left(\epsilon \alpha^{2}\right)$ for the $\mathrm{cKdV}$ model. In the present study, we assume weak forcings at the same order for both models. Accordingly, the temporal and spatial variations of channel shape are so gentle that in the boundary-value problem $\phi_{2}$ they are of $O(\alpha)$ so that their derivatives can be neglected, leaving such variations represented parametrically by the variable coefficients remaining in these equations. With this simplification, the boundary-value problem of $\phi_{2}$ (cf. TW1, equations (39)-(42)) reads

$$
\begin{gathered}
\phi_{2}(x, y, z, t)=-\frac{1}{\epsilon \alpha} \Psi(y, z ; x, t) \bar{u}_{x}, \\
\Psi_{y y}+\Psi_{z z}=1, \\
\left.\Psi_{z}\right|_{z=0}=c^{2}=\tilde{h}(x, t), \\
\Psi_{n}=0,
\end{gathered}
$$

where $\Psi_{n}=n \cdot \nabla \Psi, n$ is the outward unit vector normal to the boundary in the $(y, z)$ plane, and the first-order approximation $\tilde{\zeta}_{t}=-c^{2} \bar{u}_{x}$ has been used. The Poisson equation (6) with boundary conditions (7) and (8) is a boundary-value problem in the $(y, z)$-plane with $x$ and $t$ appearing as parameters. Here, the temporal variations of $\tilde{h}$ and $b$ in (5)-(8) are assumed one order higher in $\alpha$ than that for the original gcB model. The net effects of this difference on numerical results, however, have invariably been found to be insignificant in all cases we have experienced, even with channel shape variations not very small. Finally, substituting (5) in (3) gives

$$
\psi(x, t)=(\bar{\Psi}-\tilde{\Psi}) \bar{u}_{x x t}
$$

after the higher-order terms involving $\Psi_{t}, \Psi_{x}$ and $\Psi_{x t}$ have been neglected based on the assumed order estimate. The result indicates that $\psi$, being proportional to $\bar{u}_{x x t}$, which is the highest derivative in (1) (4), is the sole term in the model equations having a dispersive effect; it represents the contributions from the cross-flow component which generally involves the effects of vertical acceleration, the departure from the hydrostatic pressure distribution, as well as the channel configuration. 


$\begin{array}{cll}\text { Cross-sectional geometry } & \text { Mean water depth } \tilde{h} & \text { Shape factor } \kappa \\ \text { Rectangular } & \tilde{h}=h & \kappa^{2}=1 \\ \text { Semicircular } & \tilde{h}=\frac{1}{4} \pi R & \kappa^{2}=1.1267 \\ \text { Parabolic } & \tilde{h}=\frac{2}{3} h_{m} & \kappa^{2}=\frac{9}{7}+\frac{1}{15}(b / \tilde{h})^{2} \\ \text { Triangular } & \tilde{h}=\frac{1}{2} h_{m} & \kappa^{2}=\frac{3}{2}+\frac{1}{8}(b / \tilde{h})^{2} \\ \text { Trapezoidal } & \tilde{h}=\frac{1}{2}\left(2 b-h_{m}\right) h_{m} / b & \kappa^{2}=1.6133\end{array}$

TABLE 1. Shape factor $\kappa$ for variable channels

The result of our calculation thus leads to the general expression

$$
\psi=-\frac{1}{3} \kappa^{2} \tilde{h}^{2} \bar{u}_{x x t}
$$

where

$$
\kappa^{2}(x, t)=\frac{3}{\tilde{h}^{2}}(\tilde{\Psi}-\bar{\Psi})
$$

which is a dimensionless quantity we call the shape factor. The gcB model (1), (2) and the $\mathrm{cKdV}$ model (4) can then be expressed in a new version as

and

$$
\begin{gathered}
(2 b \tilde{\zeta})_{t}+\left[\left(2 b \tilde{h}-A_{d}+2 b \tilde{\zeta}\right) \bar{u}\right]_{x}=\left(A_{d}\right)_{t}, \\
\bar{u}_{t}+\bar{u} \bar{u}_{x}+\tilde{\zeta}_{x}-\frac{1}{3} \kappa^{2} \tilde{h}^{2} \bar{u}_{x x t}=-\tilde{p}_{a x},
\end{gathered}
$$

$$
\frac{1}{c_{0}} \tilde{\zeta}_{t}+\left(1+\frac{3 \tilde{\zeta}}{2 \tilde{h}_{0}}\right) \tilde{\zeta}_{x}+\frac{1}{6} \kappa^{2} \tilde{h}_{0}^{2} \tilde{\zeta}_{x x x}+\left(\frac{\tilde{h}_{0 x}}{4 \tilde{h}_{0}}+\frac{b_{0 x}}{2 b_{0}}\right) \tilde{\zeta}=-\frac{1}{2}\left(\frac{1}{2 b_{0}} A_{d}+\tilde{p}_{a}\right)_{x}
$$

Comparing with the original gcB model (TW1), we note that the result (10), (11) for $\psi$ is simplified, while the other terms in (12)-(14) remain unaltered. In $\S 3$, through numerical computations for waves travelling in variable channels, numerical results will be obtained for several representative cases to show that the difference between the two versions of the model equations is insignificant.

After the gcB model (12), (13) and cKdV equation (14) are solved to give results for the averaged flow quantities at each channel section, further three-dimensional distributions of wave patterns, such as variations of wave elevation across the channel, can be determined to second order by using the perturbation expansion and applying the unsteady Bernoulli equation at the free surface as follows:

$$
\begin{gathered}
\phi(x, y, z, t)=\alpha \phi_{1}(x, t)+\alpha^{2} \phi_{2}(x, y, z, t)+O\left(\alpha^{3}\right), \\
\zeta(x, y, t)=-\left.\phi_{t}\right|_{z=0}-\left.\frac{1}{2} \phi_{x}^{2}\right|_{z=0}+O\left(\alpha^{3}\right) .
\end{gathered}
$$

For simplicity, we shall omit the bar and tilde for the averaged quantities $\tilde{\zeta}, \bar{u}$ and $\tilde{p}_{a}$ in what follows.

\subsection{Cross-flows for some specific channel shapes}

Following similar procedures to those in TW1, we can determine $\Psi$ and the shape factor $\kappa$ for four variable channel shapes for which closed-form solutions to the Neumann problem of the Poisson equation (6)-(8) exist. The results for $\kappa$ are listed in table 1. The numerical solution of $\kappa$ for a trapezoidal channel is also given. In table 1, $h_{m}$ denotes the maximum depth and $2 b$ is the channel width at the surface. For the trapezoidal cross-section with surface width $2 b_{s}=6.25$, bottom width $2 b_{b}=3.75$ and the sidewall vertex angle of $45^{\circ}$, for which the closed-form solution is difficult to find, we have applied the Gauss-Seidel iteration method to solve (6)-(8) for $\Psi$ numerically. The resulting value for $\kappa^{2}$ for this specific shape is 1.6133 . 


\subsection{Solitary waves in uniform channels}

For uniform channels without external forcing, i.e. $p_{a}=A_{d}=0, b=$ constant and $h=$ constant $=1$, both models possess stationary solitary wave solutions. For the cKdV model, the exact solution, which is to be used later, is

with

$$
\zeta(x, t ; \kappa)=\alpha \operatorname{sech}^{2}[k(x-c t)],
$$

$$
k=\left(3 \alpha / 4 \kappa^{2}\right)^{\frac{1}{2}}, \quad c=1+\frac{1}{2} \alpha .
$$

To the same order of accuracy, the stationary solution of the $\mathrm{gcB}$ model conventionally takes the same form as (25) but with $c=(1+\alpha)^{\frac{1}{2}}$.

The exact solution to the $\mathrm{gcB}$ model for $v=u / c$ for a uniform channel (see TW1) is given by

$$
\frac{x-c t}{\kappa}= \pm \int_{v_{c}}^{v} \frac{\mathrm{d} v}{[G(v ; c)]^{\frac{1}{2}}}
$$

with

$$
\begin{gathered}
G(v ; c)=-v^{3}+3 v^{2}+\frac{6}{c^{2}}[v+\ln (1-v)], \\
c^{2}=-6\left[v_{c}+\ln \left(1-v_{c}\right)\right] / v_{c}^{2}\left(3-v_{c}\right),
\end{gathered}
$$

and the stationary solution for $\zeta$ is given by

$$
\zeta=v /(1-v) \text {. }
$$

If a wave initially has the form of (17) or (22), then it will travel in a uniform channel - rectangular or non-rectangular - permanently in form and constant in speed.

These solitary wave solutions of the two models are consistent with each other to the second order, and the discrepancies between them are insignificant when the wave amplitude is small, say $0<\alpha<0.3$.

\section{Comparison between the original and new versions of the $\mathrm{gcB}$ model}

Before we proceed to make specific comparisons between the $\mathrm{gcB}$ and $\mathrm{cKdV}$ models for variable channels, it is necessary first to establish the validity of the simplified version of the $\mathrm{gcB}$ model so that this efficient and reliable new version can be confidently applied henceforth. Here we will provide numerical results to exhibit quantitatively the differences between the two versions of the $\mathrm{gB}$ model.

As a typical case of a variable channel, we choose the parabolic cross-section for which the leading-order solution of $\psi$ for the case of a weakly varying channel is given by

$$
\psi(x, t)=-\frac{1}{3} \kappa^{2} \tilde{h}^{2} u_{x x t}, \quad \kappa^{2}=\frac{9}{7}+\frac{1}{15}(b / \tilde{h})^{2},
$$

whereas the full solution for $\psi$ is obtained (see TW1) as

$$
\psi(x, t)=\left(\frac{6 \tilde{h}}{35}-\frac{b^{2}}{45}\right) u_{x x t}-\frac{3 \tilde{h}}{10}\left(3 \mathrm{D} \tilde{h}+2 \tilde{h} u_{x}\right)_{x t}-\left(\frac{3 \tilde{h}}{35}+\frac{b^{2}}{45}\right) \mu_{x t}+\frac{3 \tilde{h}}{10}(\tilde{h} \mu)_{x t},
$$

where $\mu=\mathrm{D} \ln \left(\tilde{h} / b^{2}\right)$ and $\mathrm{D} \equiv \partial_{t}+u \partial_{x}$. For a channel fixed in time, $h_{t}=b_{t}=0$.

The two versions of the gcB model without forcing, i.e. (12), (13) with (23) and (24) and with $A_{d}=p_{a}=0$, were solved numerically by using finite difference method with an implicit predictor-corrector scheme. This scheme has second-order accuracy in space and time, and is conditionally stable (Teng 1990). We first test our scheme on the 

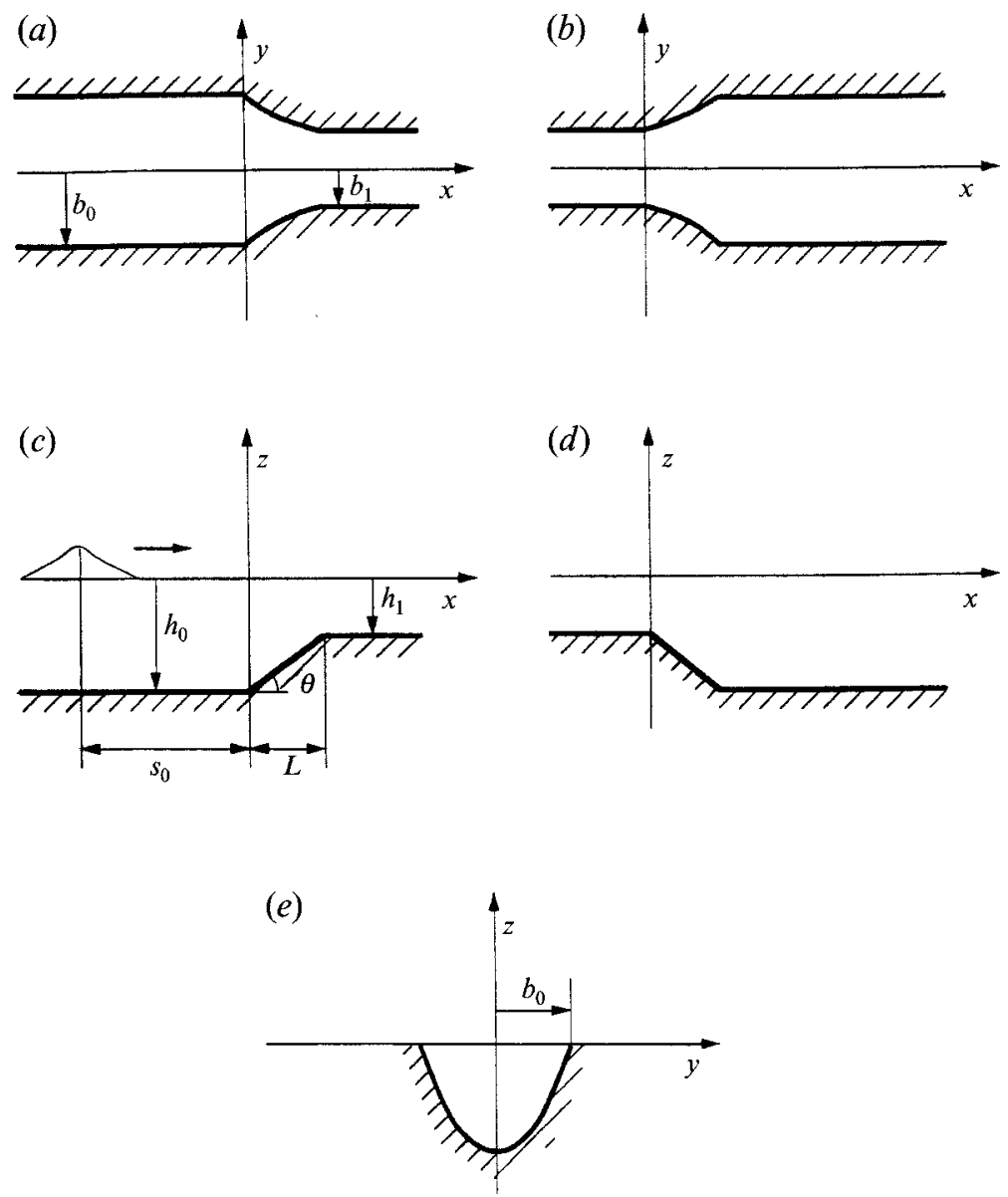

FIGURE 1. Geometric configuration of a variable parabolic channel: (a) top view of a convergent channel, $(b)$ top view of a divergent channel, $(c)$ side view of a shelf, $(d)$ side view of a step down and (e) end view of the cross-sectional geometry.

permanent-wave solution (19)-(22) of the gcB model for the uniform parabolic channel. For solitary waves of amplitude $\alpha=0.1$ travelling over a distance of about 100 water depths, the change in wave amplitude of the numerical results is less than $1 \%$ for the adopted numerical scheme.

We then applied the two versions of the $\mathrm{gcB}$ model to study the evolution of a solitary wave travelling in a variable parabolic channel with initial wave amplitude $\alpha_{0}$, water depth $h_{0}$ and channel half-width $b_{0}$. The final water depth and channel half-width are denoted by $h_{1}$ and $b_{1}$, as shown in figure 1 . These channels consist of a uniform entrance section of length 150 with constant depth $h_{0}$ and constant half-width $b_{0}$, a variable transient section of length $L$ and a uniform exit section with constant depth $h_{1}$ and half-width $b_{1}$. The variable transient section may have either a width change such as for a convergent channel (figure $1 a$ ) or a divergent channel (figure $1 b$ ), or a depth change such as a shelf up (figure $1 c$ ) or a step down (figure $1 d$ ). The initial solitary wave travels to the right starting from the initial position at $x=s_{0}=-30$.

Four cases are used in the prediction of waves: a convergent channel with $b_{1} / b_{0}=$ 0.1 ; a divergent channel with $b_{1} / b_{0}=10$, running up a shelf with $h_{1} / h_{0}=0.5$ and running down a step with $h_{1} / h_{0}=2.0$. Numerical results of the transmitted waves at $t=100$ for these cases are plotted in figure $2(a-d)$. The maximum difference between 

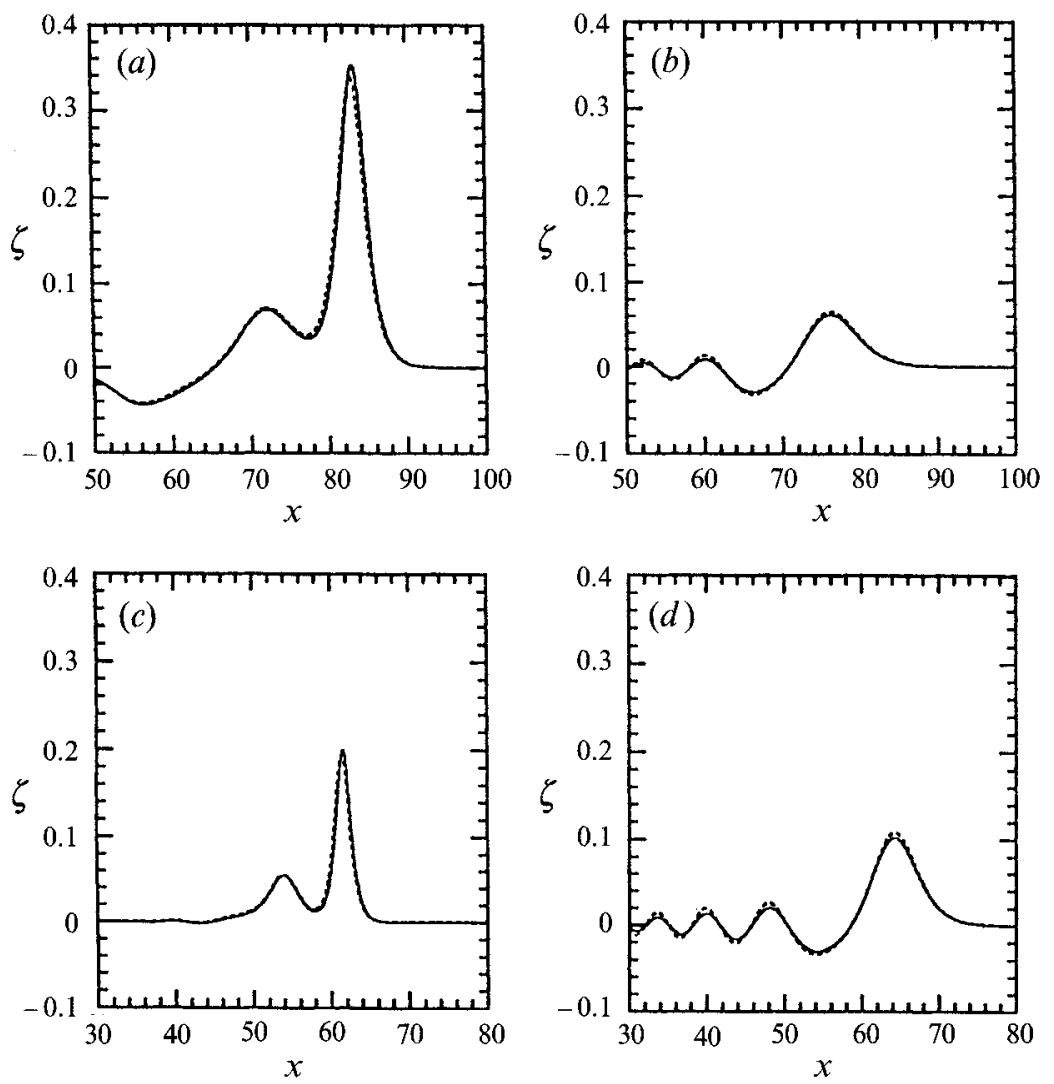

FIGURE 2. Comparison between the leading-order solution $(-)$ and the full solution (--.---) of the cross-flow problem $\phi_{2}$ for the gcB model for waves travelling in water with: $(a) h(x)=1.0, b_{0}=$ $1.5, b_{1} / b_{0}=0.1 ;(b) h(x)=1.0, b_{0}=0.15, b_{1} / b_{0}=10 ;(c) b(x)=1.5, h_{0}=1.0, h_{1} / h_{0}=0.5 ;$ and $(d)$ $b(x)=1.5, h_{0}=0.5, h_{1} / h_{0}=2$.

the two versions of the gcB model is less than $4.9 \%$, including the case of very steep change in channel width, i.e. $b_{1} / b_{0}=10$ over a length of 22.5 water depths. For waves travelling in a convergent channel with moderate width change (results not shown in figure 2), e.g. $b_{1} / b_{0}=2$ over 20 water depths, the difference between the two solutions is only about $0.9 \%$. These numerical results therefore validate the analysis presented in $\S 2$ for the simplifying assumption introduced for the $\mathrm{gcB}$ model.

\section{Conservation of mass and energy}

Here we consider the conservation properties of the $\mathrm{gcB}$ and $\mathrm{cKdV}$ models for waves propagating in a spatially varying but temporally fixed channel, and in the absence of forcing, so that in (12) (14)

$$
b=b(x), \quad h=h(x), \quad A_{d} \equiv 0, \quad p_{a} \equiv 0 .
$$

Upon integrating (12) from $x=-\infty$ to $+\infty$ under condition (25) and assuming the flow field to remain at rest at $x= \pm \infty$, we find that the $\mathrm{gcB}$ model conserves the excess mass $M_{e}$, exactly, since

$$
\frac{\mathrm{d} M_{e}}{\mathrm{~d} t}=\frac{\mathrm{d}}{\mathrm{d} t} \int_{-\infty}^{+\infty} 2 b \zeta \mathrm{d} x=0
$$


For the cKdV model, the integral of (14) over the $x$-domain under condition (25) yields

$$
\frac{\mathrm{d} M_{e}}{\mathrm{~d} t}=\int_{-\infty}^{+\infty}\left(b \tilde{h}^{\frac{1}{2}}\right)_{x} \zeta \mathrm{d} x+O\left(\alpha^{3}\right)
$$

which shows that the $\mathrm{cKdV}$ model conserves mass only for uniform channels, or for a special class of variable channels with $b h^{\frac{1}{2}}=$ constant. In general, the cKdV model does not conserve mass and the error is of $O\left(\alpha^{2}\right)$, as found by Miles (1979) in his analysis of Shuto's (1974) KdV equation.

The total flow energy is defined for the $\mathrm{gcB}$ model as

$$
E_{B}=\int_{-\infty}^{+\infty} b\left(\tilde{h} u^{2}+\zeta^{2}\right) \mathrm{d} x
$$

and for the $\mathrm{cKdV}$ model as

$$
E_{K}=\int_{-\infty}^{+\infty} 2 b \zeta^{2} \mathrm{~d} x=E_{B}+O\left(\alpha^{2} \epsilon\right)
$$

Multiplying (12) by $\zeta$ and (13) by bru $u$, we obtain from their sum and (25) that

$$
\frac{\mathrm{d} E_{B}}{\mathrm{~d} t}=\int_{-\infty}^{+\infty}\left[b u\left(\zeta^{2}\right)_{x}+\frac{2}{3}(b \tilde{h})_{x} u^{3}+\frac{2}{3} \kappa^{2} b h^{3} u u_{x x t}\right] \mathrm{d} x=O\left(\alpha^{3}\right),
$$

since $E_{B}=O\left(\alpha^{2} / \epsilon\right), u\left(\zeta^{2}\right)_{x}=O\left(\alpha^{3} \epsilon\right), \quad(b \tilde{h})_{x} u^{3}=O\left(\alpha^{4} \epsilon\right)$ and $u u_{x x t}=O\left(\alpha^{2} \varepsilon^{3}\right)$. This indicates that the $\mathrm{gcB}$ model is adiabatically invariant in energy conservation, with a small error of $O(\epsilon \alpha)$ relative to $E_{B}$. To analyse the energy conservation by the $\mathrm{cKdV}$ model, we multiply (14) with $2 b \zeta$ and integrate the product over $x$, giving, under (25),

$$
\frac{\mathrm{d} E_{K}}{\mathrm{~d} t}=2 \int_{-\infty}^{+\infty}\left[\left(\frac{b}{\tilde{h}^{\frac{1}{2}}}\right)_{x} \zeta^{3}-\frac{1}{3} \kappa^{2} b \tilde{h}^{5 / 2} \zeta \zeta_{x x x}\right] \mathrm{d} x=O\left(\alpha^{3}\right),
$$

since here we have $\left(b / \tilde{h}^{\frac{1}{2}}\right)_{x} \zeta^{3}=O\left(\alpha^{4} \epsilon\right)$ and $\zeta \zeta_{x x x}=O\left(\alpha^{2} \epsilon^{3}\right)=O\left(\alpha^{3} \epsilon\right)$. This shows that the $\mathrm{cKdV}$ model is also adiabatically invariant in conserving energy.

Thus, both of the models conserve energy at a rate with an error of $O\left(\alpha^{3}\right)$. The gcB model conserves mass exactly whereas the cKdV model has an error of $O\left(\alpha^{2}\right)$ in conserving mass.

\section{Numerical results}

We present below some typical numerical results for comparison between the $\mathrm{gcB}$ model (12), (13) and the cKdV model (14), both without forcing excitations. The same numerical scheme as described in $\S 3$ was employed for the $\mathrm{gcB}$ model, whereas for the cKdV equation (14), a simple explicit finite difference scheme was used. This explicit scheme was also tested on the exact solution (17), (18) and the results showed a relative error of less than $1 \%$.

The first case we computed is a solitary wave of initial amplitude $\alpha_{0}=0.1$ travelling in a convergent channel with $h(x)=$ constant, $b_{0}=1.5$ and $b_{1}=0.15$. The variable section is of length $L=22.5$ with half-width $b(x)$ decreasing according to $b(x)=$ $3.75 /(x+2.5)$. In this example the total width variation is somewhat extreme compared to other cases computed (see e.g. Teng \& Wu 1991), but selected as a representative case to render the boundary effects conspicuous. The numerical results for this case are shown in figures $3-5$. The elevation of the transmitted wave increases to $\alpha=0.352$ by 

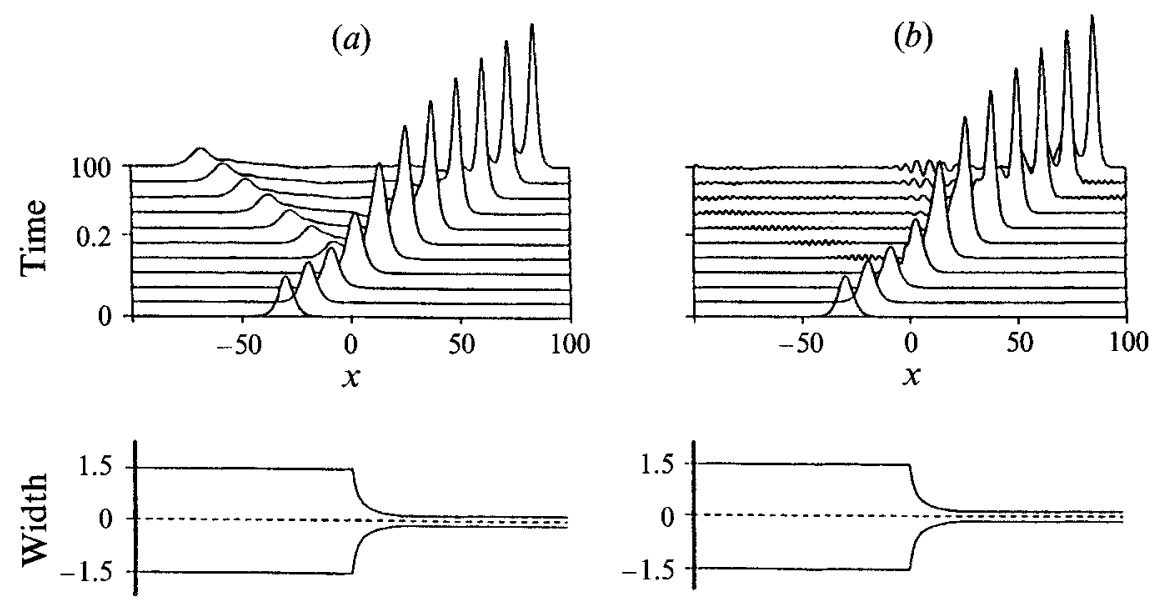

FIGURE 3. Time record of the development of a solitary wave of initial amplitude $\alpha_{0}=0.1$ in a convergent channel with $h(x)=1.0, b_{0}=1.5$ and $b_{1} / b_{0}=0.1:(a) \mathrm{gcB},(b) \mathrm{cKdV}$.
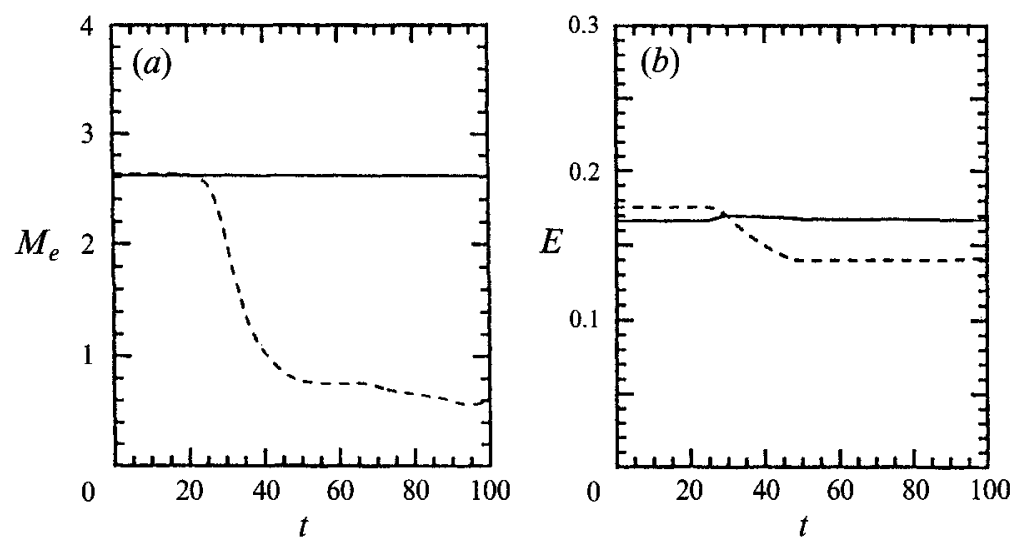

Figure 4. Conservation of (a) excess mass $M_{e}$ and $(b)$ total wave energy $E$ ( $E_{B}$ for gcB and $E_{K}$ for $\mathrm{cKdV})$, corresponding to the case shown in figure $3:-, \mathrm{gcB} ;---, \mathrm{cKdV}$.

the gcB model and to $\alpha=0.367$ by the $\mathrm{cKdV}$ model at time $t=100$, reaching the position of about $x=85$. The reflected wave predicted by the gcB model attains a maximum height of 0.043 over a length of 58.3 , receding with the critical speed of $c_{0}=1$, whereas the $\mathrm{cKdV}$ model shows, of course, no reflection. The $\mathrm{cKdV}$ model loses $78.5 \%$ excess mass and $20.3 \%$ of its energy. In contrast, the maximum error in energy conservation by the $\mathrm{gcB}$ model is as low as $2.1 \%$. However, even with such large discrepancies in mass and energy conservation, the two wave models nevertheless give quite consistent predictions for the transmitted wave with a difference of only $4.4 \%$ in wave amplitude as shown in figure 5 , the phase difference between them being primarily due to the different intrinsic wave velocities of the two models as delineated in TW1.

For a solitary wave travelling in the divergent channel, taken geometrically as an exact backward reversion of the convergent channel considered in the first case, the elevation of the transmitted wave is predicted to decrease from $\alpha_{0}=0.35$ to $\alpha=0.061$ by the gcB model and to $\alpha=0.085$ by the $\mathrm{cKdV}$ model. A train of reflected waves with negative amplitude is predicted by the gcB model, as shown in figure $6(a)$. In this case the $\mathrm{cKdV}$ gains an $256.6 \%$ extra mass and $16.6 \%$ energy, whereas the gcB conserves the mass and loses $4.4 \%$ energy. The discrepancies between the two models become 


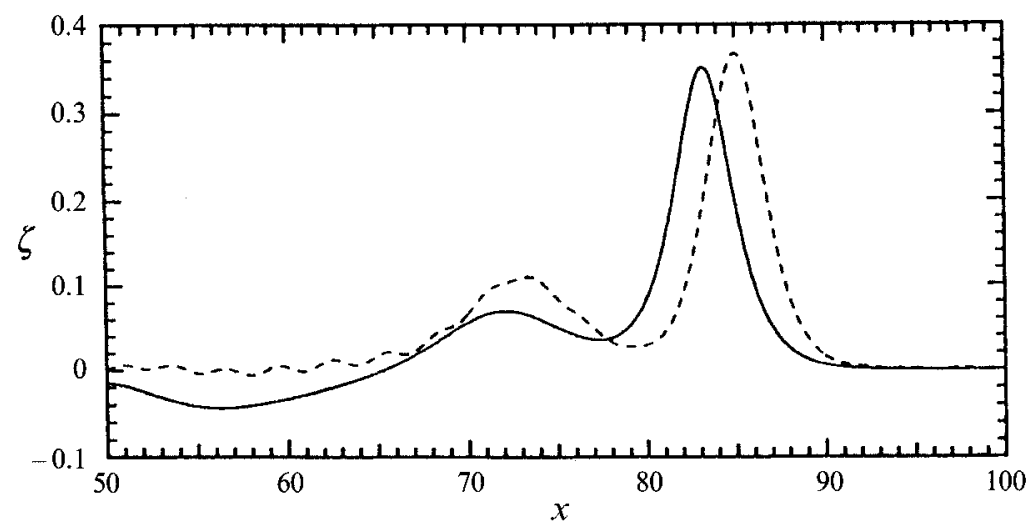

Figure 5. Comparison of wave elevation between the gcB (-) and $\mathrm{cKdV}(---)$ models. The wave elevation is computed at $t=100$ for the convergent channel shown in figure 3 .

(a)

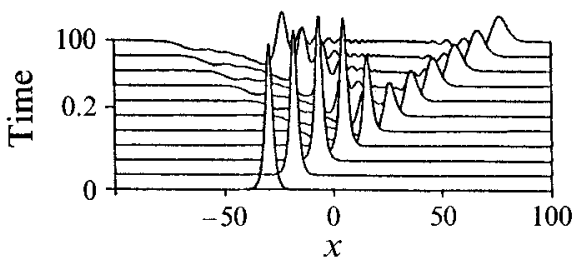

(b)
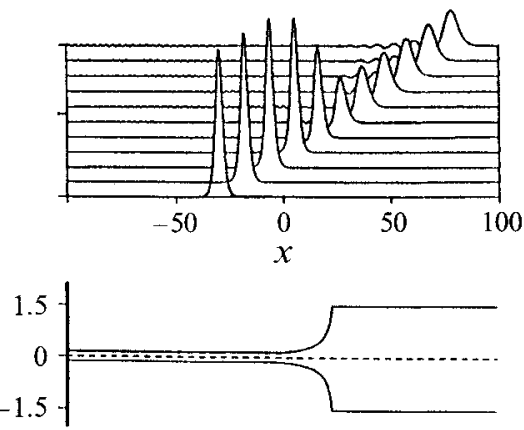

FIGURE 6. Time record of the development of a solitary wave of initial amplitude $\alpha_{0}=0.35$ in a divergent channel with $h(x)=1.0, b_{0}=0.15$ and $b_{1} / b_{0}=10:(a) \mathrm{gcB},(b) \mathrm{cKdV}$.
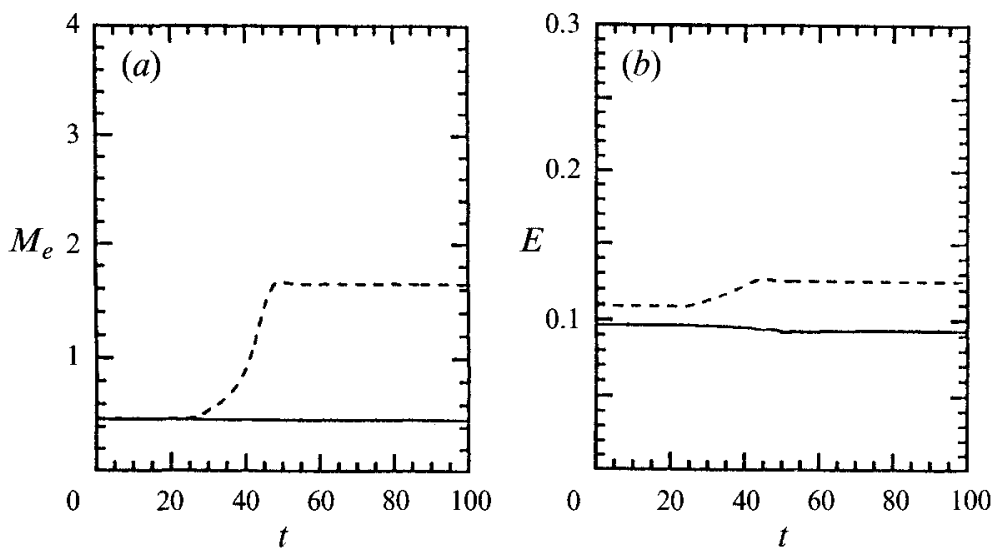

FIGURE 7. Conservation of $(a)$ excess mass $M_{e}$ and $(b)$ total wave energy $E$ ( $E_{B}$ for $\mathrm{gcB}$ and $E_{K}$ for $\mathrm{cKdV}$ ), corresponding to the case shown in figure $6:-, \mathrm{gcB} ;-\cdots, \mathrm{cKdV}$.

quite large insofar as reflected waves and energy conservation are concerned. However, for the transmitted waves, the two models still give qualitatively consistent predictions, as shown by the results in figures $6-8$.

To examine the effect of depth change, we study the evolution of a solitary wave 


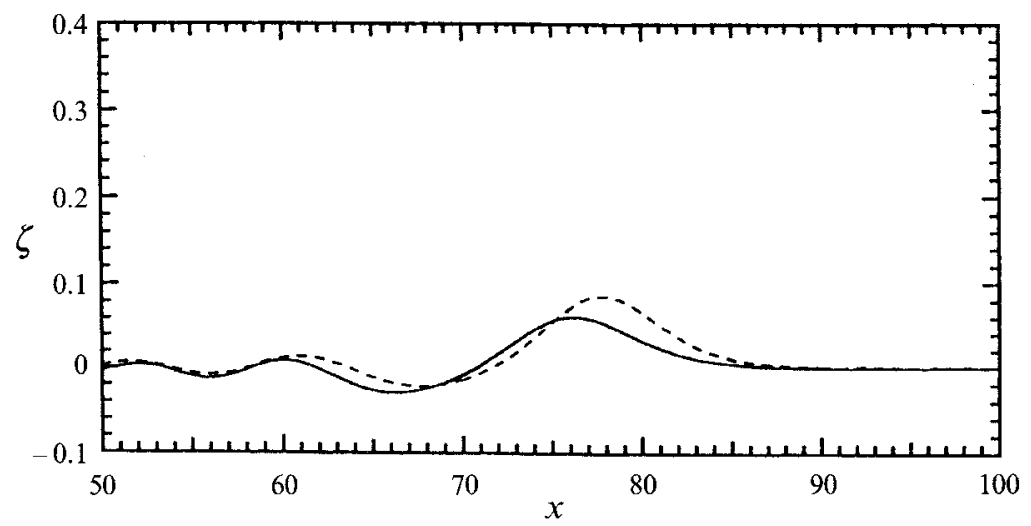

FIGURE 8. Comparison of wave elevation between the gcB (- - and $\mathrm{cKdV}(--)$ models. The wave elevation is computed at $t=100$ for the divergent channel shown in figure 6 .

(a)
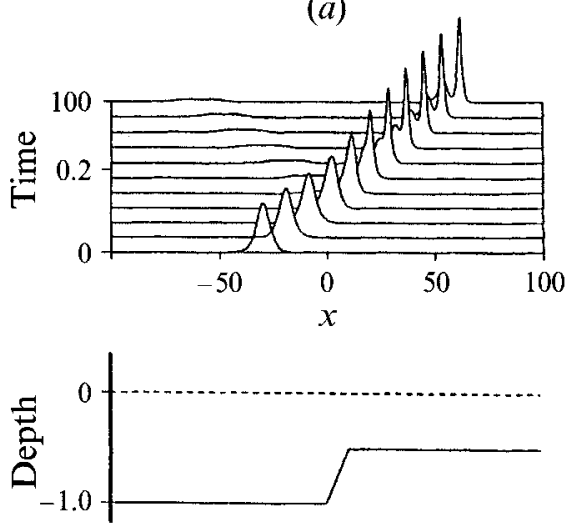

(b)
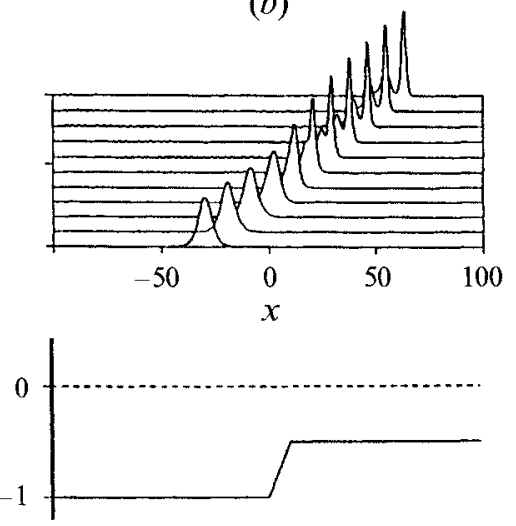

FIGURE 9. Time record of the development of a solitary wave of initial amplitude $\alpha_{0}=0.12$ running up a shelf with $b(x)=1.5, h_{0}=1.0$ and $h_{1} / h_{0}=0.5:(a) \mathrm{gcB},(b) \mathrm{cKdV}$.

running up a shelf in a parabolic channel with $b(x)=$ constant and linear depth decrease from $h_{0}=1.0$ to $h_{1}=0.5$. Results for this case are presented in figures 9-11. After running up the shelf, the original solitary wave of amplitude $\alpha_{0}=0.12$ starting from $x=-30$ at $t=0$ fissions into two waves, with the amplitude of the leading wave increased to $\alpha=0.199$ by the gcB and to $\alpha=0.203$ by the $\mathrm{cKdV}$. This fission of the solitary wave is consistent with that reported by Madsen \& Mei (1969) for waves running up a shelf in a one-dimensional or rectangular channel. The reflected wave of positive elevation predicted by the gcB model has a maximum amplitude of 0.007 and length 36.2 . Here the $\mathrm{cKdV}$ model terminally loses $12.9 \%$ mass and gains $8.6 \%$ energy at $t=100$, whereas the gcB model loses $2.2 \%$ energy as indicated in figure 10 . As in the previous cases, these errors in fulfilling conservation laws have little effect on the validity of the cKdV model in predicting the transmitted waves. For the present case, the difference between the two models in wave elevation for the leading wave is $2.0 \%$.

The discrepancies in wave speed between the two wave models, as exhibited in the comparative figures, are caused not only by variations in depth and width, but also by the fact that even for uniform channels the two models possess slightly different solitary wave solutions, with the gcB solitary wave propagating more slowly for waves 

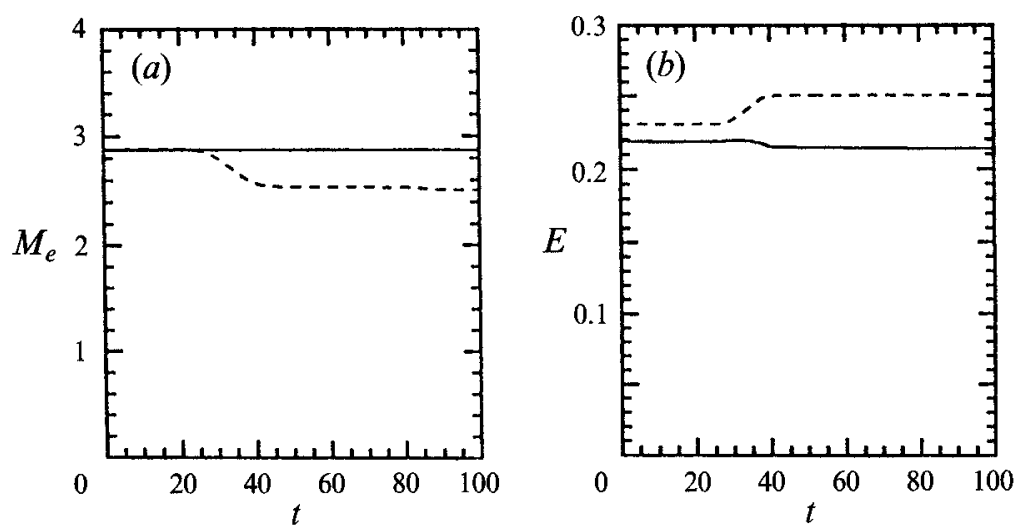

FIGURE 10. Conservation of (a) excess mass $M_{e}$ and $(b)$ total wave energy $E$ ( $E_{B}$ for gcB and $E_{K}$ for $\mathrm{cKdV})$, corresponding to the case shown in figure 9:-_, $\mathrm{gcB}:---, \mathrm{cKdV}$.

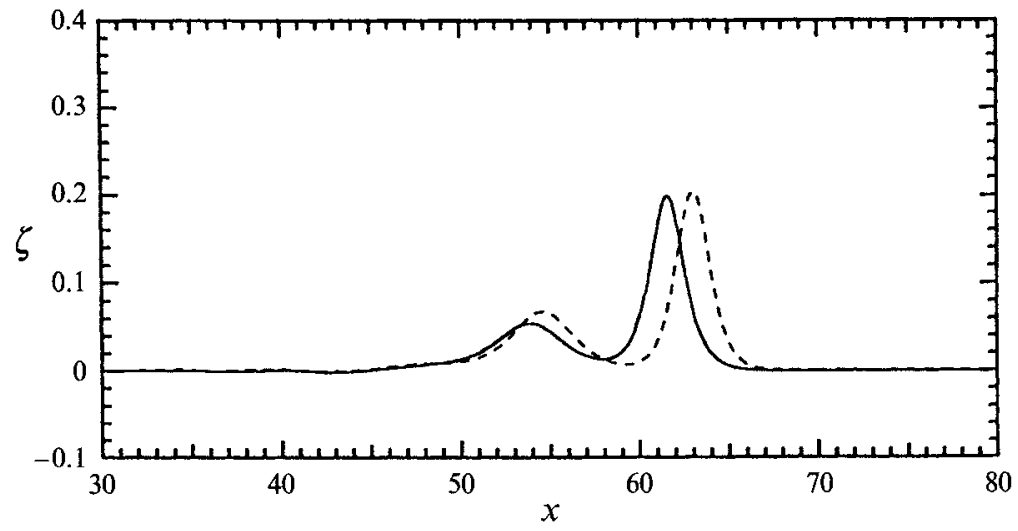

FIGURE 11. Comparison of wave elevation between the gcB (-) and cKdV (--) models. The wave elevation is computed at $t=100$ for the corresponding case shown in figure 9 .

of equal height (TW1). For the same reason, the initial values of the excess mass and wave energy also have slightly different values for the different wave models.

Another result of interest is that even though theoretically both models adiabatically conserve energy to the third order, the numerical results show that invariably the $\mathrm{cKdV}$ model has a considerably poorer accuracy in conserving energy, the error being found sometimes as large as more than $20 \%$ versus gcB's $2 \%$.

\section{Comparison with experimental data}

In this section, we compare our numerical results with the experimental data obtained by Chang et al. (1979) who measured the elevation of the transmitted waves in both a divergent and a convergent rectangular channel with uniform depth. The divergent channel has an initial half-width $b_{0} / h=0.25$ and then gradually and linearly expands to $b_{1} / h=2.5$ over a distance of $L / h=120$. In their presentation for the divergent channel, the position $x^{\prime}$ was measured from $2.63 \mathrm{~m}$ (water depth $20 \mathrm{~cm}$ ) ahead of the channel vertex, which corresponds to our $x=-13.1$. Three sets of experimental data were obtained, for $\alpha_{0}=0.088,0.185$ and 0.259 . The convergent channel has the initial half-width $b_{0} / h=1.5$ and converges to $b_{1} / h=0$ over a length of $L / h=80$. In 

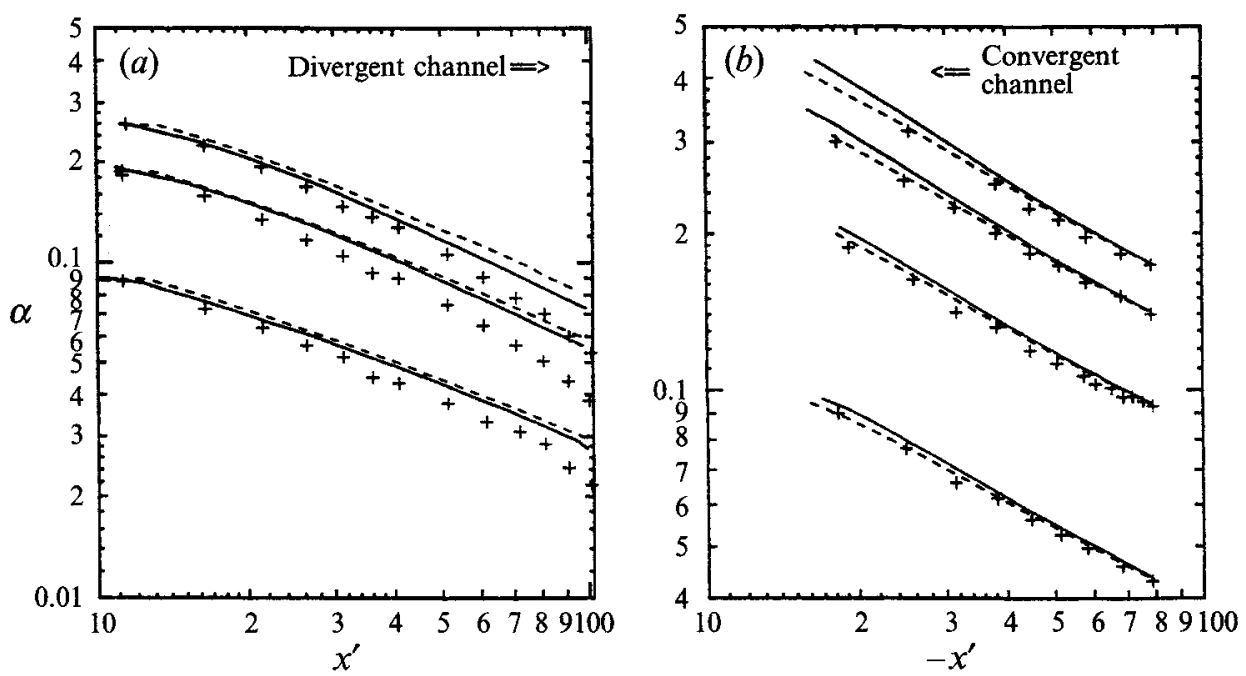

FIGURE 12. Comparison between numerical results and Chang et al.'s (1979) experimental data for transmitted waves in $(a)$ a divergent channel of expansion angle 0.019 rad, with initial amplitudes $\alpha_{0}=0.088,0.185,0.259 ;(b)$ a convergent channel of convergence angle $0.019 \mathrm{rad}$, with initial amplitudes $\alpha_{0}=0.043,0.093,0.140,0.174$. + , experiment; - - gcB; ---, cKdV.

Chang et al.'s (1979) original report, the convergent case was considered as an inverse of the divergent case, and the waves in the convergent channel were imagined to travel in the negative direction in the divergent channel. Their $x^{\prime}$ is thus denoted negative and $x^{\prime}=-80$ corresponds to the channel vertex where the width starts to decrease. Four sets of data were measured for the convergent channel, with initial amplitudes $\alpha_{0}=$ $0.043,0.093,0.140$ and 0.174 . In the present study, we carried out numerical simulations based on both the $\mathrm{gcB}$ and $\mathrm{cKdV}$ models for comparison with these experimental results. Our numerical results, obtained with $\kappa=1$ for the rectangular channel, are plotted in figure 12. In order to match with the original plotting of Chang et al., we converted our results to the $x^{\prime}$ coordinate. These results show that our two models give very consistent predictions for the height of the transmitted waves. They also agree well with the experimental data for a convergent channel, with the inviscid $\mathrm{cKdV}$ model providing slightly better results. For waves in the divergent channel, in the first half of the channel, the two models give quite good predictions of the experimental results, while near the exit, the waves measured in the experiment seem to decay much faster. This difference may be caused by the neglected dissipation effects, as argued in Chang et al.'s original paper. The horizontal and vertical coordinates in figure 12 are in a log scale. In figure $12(b)$ for the convergent channel, the data lines are seen to be quite straight, which indicates the existence of a power law, i.e. $\alpha \sim b^{m}$, where $m$ need not be an integer. However, it is difficult to draw a conclusion from figure 12(a) for the divergent channel. (For power laws, see Chang et al. 1979 and Miles 1979).

\section{Discussion}

First we point out the good agreement between our results from the gcB model (figure $12 a, b$ ) and Kirby \& Vengayil's (their figures 3,5 and 6) coupled two-way KdV equations, which verifies that both theories share a comparable high accuracy. This is expected, since the difference between the two lies only in that the reflected waves are assumed linear for the coupled $\mathrm{KdV}$ equations. The numerical efficiency of the $\mathrm{gcB}$ 
model is probably slightly better than the coupled $\mathrm{KdV}$ model. Both of these models are a system of two equations for two unknowns; however, the $\mathrm{gcB}$ model solves the wave elevation (including both transmitted and reflected) and fluid velocity simultaneously, whereas the coupled $\mathrm{KdV}$ equation gives only the wave elevation.

Another point we should address is that for our models we have assumed that the channel width and the water depth are of the same order. For wide channels, Mathew \& Akylas (1990) derived a K-P equation for long waves moving in a wide uniform trapezoidal channel. A further study to compare our wave models, which may require modifications for wide channels, with Mathew \& Akylas' K-P equation would be useful.

\section{Conclusion}

The $\mathrm{gcB}$ channel wave model has been further simplified in the present study. Using the new version of the $\mathrm{gcB}$ model can significantly reduce the computational effort in numerical simulations and can also reveal more clearly the underlying physical effects of channel cross-section and variations of wave generation and propagation. From our numerical comparisons given above, the simplified $\mathrm{gcB}$ model is found to preserve the same accuracy as the original model.

The $\mathrm{gcB}$ model permits propagation of long waves in both directions along a variable channel and conserves mass exactly. The cKdV model, being derived for a unidirectional wave only, does not conserve mass owing to its neglect of reflected waves in variable channels. Our numerical simulations for various cases have shown that regardless of how poorly the $\mathrm{cKdV}$ model conserves mass (e.g. gaining $200 \%$ of its original excess mass), it still provides quite consistent results for the transmitted waves, in good agreement with the $\mathrm{gcB}$ model. Our numerical results for both models show good agreement with the experiments of Chang et al. (1979) for waves transmitted along a divergent or convergent channel.

This work was partly supported by NSF Grant 4DMS-8901440 and its preceding NSF Grant MSM-8706045. We are indebted to the referees for some interesting discussions on numerical results in comparison with the paper by Kirby \& Vengayil.

\section{REFERENCES}

Chang, P., Melville, W. K. \& Miles, J. W. 1979 On the evolution of a solitary wave in a gradually varying channel. J. Fluid Mech. 95, 401-414.

Chol, H.-S., BAI, K. J., KIM, J.-W. \& CHо, I.-H. 1990 Nonlinear free surface waves due to a ship moving near the critical speed in a shallow water. In 18th Symp. on Naval Hydrodynamics, Aug. 12-24, Ann Arbor, MI, pp. 173-190. Washington: National Academy Press.

Chwang, A. T. \& Wu, T. Y. 1976 Cylindrical solitary waves. In Waves on Water of Variable Depth (ed. D. G. Provis \& R. Radok), Lecture Notes in Physics, vol. 64, pp. 80-90. Springer.

Ertekin, R. C. \& Wehausen, J. V. 1987 Some solition calculations. In Proc. 16th Symp. on Naval Hydrodynamics (ed. W. C. Webster), pp. 167-184. Washington: National Academy Press.

KIRBY, J. T. \& VENGAYIL, P. 1988 Nonresonant and resonant reflection of long waves in varying channels. J. Geophy. Res. 93, 10782-10796.

LIU, P. L.-F., Yoon, S. B. \& KIRBY, J. T. 1985 Nonlinear refraction-diffraction of waves in shallow water. J. Fluid Mech. 153, 185-201.

MAdSEN, O. S. \& MeI, C. C. 1969 The transformation of a solitary wave over an uneven bottom. J. Fluid Mech. 39, 781-791.

MATHEW, J. \& AKYLAS, T. R. 1990 On three-dimensional long water waves in a channel with sloping sidewalls. J. Fluid Mech. 215, 289-307. 
Miles, J. W. 1979 On the Korteweg-de Vries equation for a gradually varying channel. $J$. Fluid Mech. 91, 181-190.

Peregrine, D. H. 1967 Long waves on a beach. J. Fluid Mech. 27, 815-827.

SCHEMBER, H. R. 1982 A new model for three-dimensional nonlinear dispersive long waves, $\mathrm{PhD}$ thesis, California Institute of Technology, Pasadena, CA.

Shuto, N. 1974 Nonlinear long waves in a channel of variable section. Coastal Engng in Japan 17, $1-12$.

TENG, M. H. 1990 Forced emissions of nonlinear water waves in channels of arbitrary shape. $\mathrm{PhD}$ thesis, California Institute of Technology, Pasadena, CA.

TENG, M. H. \& WU, T. Y. 1990 Generation and propagation of nonlinear water waves in a channel with variable cross section. In Engineering Science, Fluid Dynamics, pp. 87-108. World Scientific.

Teng, M. H. \& WU, T. Y. 1991 Run-up of tsunami waves in coastal water of variable depth and width. In Proc. of Intl Workshop on Technology for Hong Kong's Infrastructure Development, pp. 529-544. Commercial Press (Hong Kong) Ltd.

TENG, M. H. \& WU, T. Y. 1992 Nonlinear water waves in channels of arbitrary shape. J. Fluid Mech. 242, 211-233 (referred to herein as TW1).

Wu, T. Y. 1979 Tsunamis-Proc. National Science Foundation Workshop, May 7-9, 1979, pp. 110-149, Pasadena: Tetra Tech. Inc.

Wu, T. Y. 1981 Long waves in ocean and coastal waters. J Engng Mech. Div. ASCE, 107, 501-522. 